\title{
WEAKLY ALMOST PERIODIC FUNCTIONS AND FOURIER-STIELTJES ALGEBRAS OF LOCALLY COMPACT GROUPS
}

\author{
BY
}

CHING CHOU

\begin{abstract}
A noncompact locally compact group $G$ is called an Eberlein group if $W(G)=B(G)^{-}$where $W(G)$ is the algebra of continuous weakly almost periodic functions on $G$ and $B(G)^{-}$is the uniform closure of the Fourier-Stieltjes algebra of $G$. We show that if $G$ is a noncompact [ $I N]$-group or a noncompact nilpotent group then $W(G) / B(G)^{-}$contains a linear isometric copy of $l^{\infty}$. In particular, $G$ is not an Eberlein group. On the other hand, finite direct products of Euclidean motion groups and, by a result of $\mathrm{W}$. Veech, noncompact semisimple analytic groups with finite centers are Eberlein groups.
\end{abstract}

1. Introduction. Let $G$ be a locally compact group, $C(G)$ the space of bounded continuous complex-valued functions on $G$ with the sup norm, $W(G)$ the space of continuous weakly almost periodic (w.a.p) functions on $G, B(G)$ the Fourier-Stieltjes algebra of $G$ and $B(G)^{-}$its uniform closure in $C(G)$. It is known that $B(G)^{-} \subset W(G)$ and if $G$ is compact then $C(G)=W(G)=B(G)^{-}$. Therefore, in this paper, $G$ is assumed to be noncompact. In his efforts to characterize w.a.p. functions, W. F. Eberlein first raised the question whether, for abelian $G, B(G)^{-}=W(G)$. W. Rudin [24] made the initial contribution to this question by showing that $B(G)^{-} \subset W(G)$ if $G$ is abelian and contains a discrete subgroup which is not of bounded order and D. Ramirez [23], by studying discrete abelian groups with bounded orders together with the structure theorem for locally compact abelian groups was able to show that Rudin's conclusion holds for all abelian groups, see the monographs [4 and 11] for complete accounts of this result.

In this paper we will extend Rudin-Ramirez' result to include many nonabelian groups. Our main result is the following.

THEOREM. Let $G$ be either a noncompact nilpotent group or a noncompact [IN]-group. Then $B(G)^{-} \subset W(G)$. In fact, the quotient Banach space $W(G) / B(G)^{-}$contains $a$ linear isometric copy of $l^{\infty}$.

Received by the editors August 17, 1981.

1980 Mathematics Subject Classification. Primary 43A60, 43A30, 43A46; Secondary 22D05, 22D10, $22 \mathrm{D} 25$.

Key words and phrases. Locally compact groups, weakly almost periodic functions, Fourier-Stieltjes algebras, unitary representations, weak Sidon sets, relatively dense sets, $C^{*}$-algebras, $[I N]$-groups, nilpotent groups, motion groups, semisimple groups, weakly almost periodic compactification. 
Recall that a locally compact group $G$ is called an $[I N]$-group if it has a compact neighborhood of the identity which is invariant under all the inner automorphisms of $G$, cf. [17]. In particular discrete groups are [IN]-groups. To prove the above theorem, we will consider discrete groups first. Since there are no satisfactory structure theorems for general discrete groups, our approach is necessarily free of any structure theorems for such groups.

Rudin-Ramirez' result cannot be extended to include all locally compact groups. Indeed, let $A P(G)$ be the space of continuous almost periodic (a.p.) functions on $G$ and $C_{0}(G)$ the space of continuous functions on $G$ which are vanishing at infinity. Then the following inclusive relations hold.

$$
A P(G) \oplus C_{0}(G) \subset B(G)^{-} \subset W(G) .
$$

As in [7], a group $G$ is said to be minimally w.a.p. if $A P(G) \oplus C_{0}(G)=W(G)$. We would like to give another definition here.

Definition. A noncompact locally compact group $G$ is called an Eberlein group if $W(G)=B(G)^{-}$.

By (1.1), if $G$ is minimally w.a.p. then it is an Eberlein group. It is known that minimally w.a.p. groups exist, cf. [5, 7 and 26]. Therefore Eberlein groups exist.

After giving some preliminary results and notations in $\S 2$, we will prove that infinite discrete groups are not Eberlein groups in \$3, the key step for proving our main result cited earlier. The notion of weak Sidon sets for discrete groups as defined by Picardello [22] will come into play. The structure theorem for [IN]-groups by Grosser and Moskowitz [17] will then be applied in $\$ 4$ to prove our main result.

The w.a.p. compactification of a locally compact group $G$ will be denoted by $G^{\omega}$, cf. [4]. In $\$ 5$ we will look at the weakly almost periodic compactifications of finite products of locally compact groups and will provide examples of Eberlein groups that are not minimally w.a.p. Among other things, we will prove that if $G$ and $H$ are noncompact [ $I N]$-groups or noncompact nilpotent groups then $(G \times H)^{\omega}$ is not homeomorphic to $G^{\omega} \times H^{\omega}$, or equivalently, $W(G) \otimes W(H)$ is not uniformly dense in $W(G \times H)$. This result seems to be new even for general abelian groups, cf. [10, p. 105; 2, p. 171 and 21, Theorem 1.3].

The inclusive relation $A P(G) \oplus C_{0}(G) \subset B(G)^{-}$will be studied in $\S 6$. By applying arguments contained in [7, Theorem 3.1] we will prove that for a connected solvable group $G$, the following conditions are equivalent: (i) $G$ is minimally w.a.p., (ii) $A P(G) \oplus C_{0}(G)=B(G)^{-}$, (iii) $G / K(G)$ is topologically isomorphic to $M(2)$ where $K(G)$ is the largest compact normal subgroup of $G$ and $M(2)$ is the Euclidean motion group of the plane.

2. Preliminaries. To a given locally compact group $G$, we will assume that a left Haar measure has been chosen for $G$. Integration with respect to this fixed left Haar measure will be denoted by $\int_{G} \cdots d x$. A continuous unitary representation of $G$ will simply be called a unitary representation of $G$. Let $\pi$ be a unitary representation of $G$ on a Hilbert space $H$. We will denote the corresponding representation of $L^{1}(G)$ 
on $H$ again by $\pi$ : for $f \in L^{1}(G), \pi(f)=\int_{G} f(x) \pi(x) d x$. On $L^{1}(G)$ we will denote the maximal $C^{*}$-norm by $\|\cdot\|_{*}$ : for $f \in L^{1}(G)$,

$$
\|f\|_{*}=\sup \{\|\pi(f)\|: \pi \text { a unitary representation of } G\} \text {. }
$$

The completion of $\left(L^{1}(G),\|\cdot\|_{*}\right)$, designated by $C^{*}(G)$, is the $C^{*}$-algebra of $G$.

Recall that $B(G)$, the Fourier-Stieltjes algebra of $G$, is the linear span of the set of all continuous positive definite functions of $G$, or equivalently, the space of coefficient functions of unitary representations of $G$. The dual Banach space of $C^{*}(G)$ can be realized as $B(G)$ : for $u \in B(G), f \in L^{1}(G),\langle u, f\rangle=\int_{G} u(x) f(x) d x$. Furthermore, with respect to the dual norm of $C^{*}(G)$ and pointwise multiplication, $B(G)$ forms a commutative Banach algebra. See Eymard [14] for all the results mentioned in this paragraph. Note that for abelian $G, B(G)=\{\hat{\mu}: \mu$ is a bounded regular Borel measure on the dual group $\hat{G}$ of $G\}$ where $\hat{\mu}$ is the Fourier-Stieltjes transform of $\mu$ and $\|\hat{\mu}\|=|\mu|(\hat{G})$.

If $f \in C(G), x \in G$ then $l_{x} f$, the left translate of $f$ by $x$, is defined by $l_{x} f(y)=$ $f\left(x^{-1} y\right)$. Recall that $f \in C(G)$ is w.a.p. if the set $\left\{l_{x} f: x \in G\right\}$ is relatively compact with respect to the weak topology of $C(G)$. The space of w.a.p. functions on $G$, $W(G)$, is a translation invariant $C^{*}$-subalgebra of $C(G)$. It is well known that $W(G)$ has a unique translation invariant mean $m_{G}$, cf. Ryll-Nardzewski [25]. Let $W_{0}(G)=$ $\left\{f \in W(G): m_{G}(|f|)=0\right\}$. Then $W(G)=A P(G) \oplus W_{0}(G)$, cf. de Leeuw and Glicksberg [9]. W.a.p. functions were first defined and studied by Eberlein [12]. Many of the basic results (for abelian groups) are due to him. Burckel's monograph [4] is a convenient reference for many of the results on w.a.p. functions mentioned throughout this paper. A very useful criterion for a function $f \in C(G)$ to be w.a.p. is given in Grothendieck [18]: $f$ is w.a.p. if and only if whenever $\left\{x_{i}\right\}$ and $\left\{y_{k}\right\}$ are two sequences in $G$ and $\lim _{i} \lim _{k} f\left(x_{i} y_{k}\right)$ and $\lim _{k} \lim _{i} f\left(x_{i} y_{k}\right)$ exist, then they are equal.

If $E$ is a subset of $C(G)$, its closure with respect to sup norm topology will be denoted by $E^{-}$. In [15, Chapitre III], Godement proved that $B(G)$ has a unique invariant mean. Since $B(G) \subset W(G)$, cf. [4], it is the restriction of $m_{G}$ to $B(G)$. He also proved that $B(G)=B_{d}(G) \oplus B_{c}(G)$ where $B_{d}(G)=B(G) \cap A P(G)=$ $\left\{\sum_{j=1}^{\infty} c_{j} \varphi_{j}: \Sigma\left|c_{j}\right|<\infty, c_{j} \in \mathbf{C}\right.$ and $\varphi_{j}$ are coefficient functions of irreducible finitedimensional unitary representations of $G\}$ and $B_{c}(G)=B(G) \cap W_{0}(G)=$ the space of coefficient functions of unitary representations of $G$ which have no nonzero finite dimensional invariant subspaces. By von Neumann's approximation theorem for a.p. functions, $B_{d}(G)^{-}=A P(G)$. Since each $f \in C_{0}(G)$ can be approximated uniformly on $G$ by the convolution of two continuous functions with compact supports, cf. [14, p. 210], one sees that $C_{0}(G) \subset B_{c}(G)^{-}$. In particular, $A P(G) \oplus C_{0}(G) \subset B(G)^{-}$ as we have claimed in (1.1).

Since $W(G)=A P(G) \oplus W_{0}(G)$, by the open mapping theorem, there exists a constant $c$ such that for any $f \in W(G)$ with $f=g+h, g \in A P(G), h \in W_{0}(G)$ we have $\|h\|_{\infty} \leqslant c\|f\|_{\infty}$. Using this fact, it is easy to see that $B_{c}(G)^{-}=B(G)^{-} \cap W_{0}(G)$. Therefore we have the following. 
LeMma 2.1. Let $G$ be a noncompact locally compact group. Then $B(G)^{-}=A P(G) \oplus$ $B_{c}(G)^{-}$. In particular, $B(G)^{-}=A P(G) \oplus C_{0}(G)$ if and only if $B_{c}(G) \subset C_{0}(G)$.

SOME NOTATIONS. If $f$ is a continuous function on a topological space, the support of $f$ will be denoted by suppt $f$. If $G$ is a group, its identity will be denoted by $e=e_{G}$. If $X$ is a set, $|X|$ denotes the cardinality of $X$.

3. Discrete groups. Throughout this section, $G$ will denote an infinite discrete group.

Definition 3.1. A subset $S$ of $G$ is called a $t$-set if $(T \cap x T) \cup(T \cap T x)$ is finite whenever $x \in G, x \neq e$.

The above definition is taken from Burckel [4]. $t$-sets were first introduced by Rudin [24] in his study of w.a.p. functions on abelian groups. The following lemma was proved by him in [24] for abelian groups; for a general group, a proof is given in [4]. For completeness, we will present a short proof here.

Lemma 3.2. Suppose that $S$ is a t-set in $G$ and $f \in C(G)$ such that suppt $f$ is contained in $S$. Then $f \in W(G)$.

Proof. According to Grothendieck's criterion, it suffices to show that whenever $\left\{x_{n}\right\}$ and $\left\{y_{m}\right\}$ are two sequence in $G$ such that

$$
L=\lim _{m} \lim _{n} f\left(x_{n} y_{m}\right), \quad R=\lim _{n} \lim _{m} f\left(x_{n} y_{m}\right)
$$

exist then $L=R$. It is easy to see that if either $\left\{x_{n}\right\}$ or $\left\{y_{m}\right\}$ is eventually a constant then $L=R$. Therefore, we only have to consider the case that $x_{n} \neq x_{n^{\prime}}$ if $n \neq n^{\prime}$ and $y_{m} \neq y_{m^{\prime}}$ if $m \neq m^{\prime}$. We claim that in this case $L=R=0$. Indeed, if $\lim _{n} f\left(x_{n} y_{m_{0}}\right)$ $\neq 0$ for some fixed $m_{0}$ then $x_{n} \in S y_{m_{0}}^{-1}$ when $n$ is large. If $m \neq m_{0}$ then $S y_{m_{0}}^{-1} \cap S y_{m}^{-1}$ is finite and hence $f\left(x_{n} y_{m}\right) \neq 0$ for only finite many $n$. So $\lim _{n} f\left(x_{n} y_{m}\right)=0$ if $m \neq m_{0}$. Therefore $L=0$. By symmetry, $R=0$.

Definition 3.3. A finite subset $C$ of $G$ is called an $n$-square if $C=A \cdot B$ where $|A|=|B|=n$ and $|C|=n^{2}$. A subset $S$ of $G$ is said to contain large squares if for each $k \in \mathbf{N}$ (the set of positive integers), $S$ contains a $k$-square.

REMARK. It is demonstrated in the proof of [20, Theorem 1.4] that whenever $S$ satisfies the (somewhat weaker) condition: $\sup \{\min (|A|,|B|): A \cdot B \subset S\}=\infty$, then it contains large squares.

If $S$ is a subset of $G$ then $l^{\prime}(S)$ can be considered as a subset of $l^{1}(G)$ : if $f \in l^{1}(S)$ then extend $f$ to a function on $G$ by setting $f(x)=0$ if $x \in G \backslash S$. Therefore, $l^{1}(S)$ is the space of functions in $l^{\prime}(G)$ with their supports contained in $S$. For abelian $G$, the following proposition in equivalent to Theorem 1.4 of López and Ross [20]. Our proof is a modification of theirs. (They attributed the proof there to S. Saeki.)

Proposition 3.4. Suppose that $S$ is a subset of $G$ such that it contains large squares. Then $\|\cdot\|_{1}$ and $\|\cdot\|_{*}$ are not equivalent on $l^{1}(S)$.

Proof. Here $\|\cdot\|_{*}$ is the $C^{*}$-norm on $l^{\prime}(G)$ as defined in $\$ 2$. For each $n \in \mathbf{N}$, choose an $n$-square $C=A \cdot B$ in $S$, say, $A=\left\{a_{1}, \ldots, a_{n}\right\}$ and $B=\left\{b_{1}, \ldots, b_{n}\right\}$. Let $\left(u_{j, k}\right)$ be an $n \times n$ unitary matrix with complex entries and with $\left|u_{j, k}\right|=1 / \sqrt{n}$ for 
each $(j, k)$. For a fixed $t \in G$, let $\delta_{t}$ be the function on $G$ which takes 1 at $t$ and zero otherwise. Let

$$
g=\sum_{j, k=1}^{n} u_{j k} \delta_{a_{j} b_{k}}
$$

Then $g \in l^{1}(S)$ and

$$
\|g\|_{1}=\sum_{j, k=1}^{n}\left|u_{j k}\right|=n^{2} \cdot \frac{1}{\sqrt{n}}=n^{1+1 / 2} .
$$

We would like to find an upper bound for $\|g\|_{*}$.

Let $\pi$ be a unitary representation of $G$ on a Hilbert space $H$. Then using the notation given in $\S 2, \pi(g)=\sum_{j, k=1}^{n} u_{j k} \pi\left(a_{j} b_{k}\right)$. For $\xi \in H$,

$$
\begin{aligned}
\|\pi(g) \xi\| & =\left\|\sum_{j=1}^{n} \pi\left(a_{j}\right)\left(\sum_{k=1}^{n} u_{j k} \pi\left(b_{k}\right) \xi\right)\right\| \leqslant \sum_{j=1}^{n}\left\|\pi\left(a_{j}\right)\right\|\left\|\sum_{k=1}^{n} u_{j k} \pi\left(b_{k}\right) \xi\right\| \\
& =\sum_{j=1}^{n}\left\|\sum_{k=1}^{n} u_{j k} \pi\left(b_{k}\right) \xi\right\| \leqslant n^{1 / 2}\left(\sum_{j=1}^{n}\left\|\sum_{k=1}^{n} u_{j k} \pi\left(b_{k}\right) \xi\right\|^{2}\right)^{1 / 2} .
\end{aligned}
$$

Now consider the Hilbert space $V=H \oplus H \oplus \cdots \oplus H$ ( $n$ copies) with norm $\left\|\left(\xi_{1}, \ldots, \xi_{n}\right)\right\|=\left(\left\|\xi_{1}\right\|^{2}+\cdots+\left\|\xi_{n}\right\|^{2}\right)^{1 / 2}$. The operator on $V$ which sends $\left(\xi_{1}, \ldots, \xi_{n}\right)$ to $\left(\sum_{k=1}^{n} u_{1 k} \xi_{k}, \sum_{k=1}^{n} u_{2 k} \xi_{k}, \ldots, \sum_{k=1}^{n} u_{n k} \xi_{k}\right)$ is clearly unitary. Therefore,

$$
\begin{aligned}
\left\|\left(\pi\left(b_{1}\right) \xi_{1}, \ldots, \pi\left(b_{n}\right) \xi_{n}\right)\right\| \\
\quad=\left\|\left(\sum_{k=1}^{n} u_{1 k} \pi\left(b_{k}\right) \xi, \sum_{k=1}^{n} u_{2 k} \pi\left(b_{k}\right) \xi, \ldots, \sum_{k=1}^{n} u_{n k} \pi\left(b_{n}\right) \xi\right)\right\|,
\end{aligned}
$$

or equivalently,

$$
\sum_{j=1}^{n}\left\|\sum_{k=1}^{n} u_{j k} \pi\left(b_{k}\right) \xi\right\|^{2}=\sum_{k=1}^{n}\left\|\pi\left(b_{k}\right) \xi\right\|^{2}=n\|\xi\|^{2} .
$$

Combining this with (3.2) we get

$$
\|\pi(g) \xi\| \leqslant n^{1 / 2}\left(n\|\xi\|^{2}\right)^{1 / 2}=n\|\xi\| .
$$

Therefore, $\|\pi(g)\| \leqslant n$, for every unitary representation $\pi$ of $G$, and hence $\|g\|_{*} \leqslant n$. Since $n$ can be arbitrarily large, comparing with (3.1), we conclude that $\|\cdot\|_{1}$ and $\|\cdot\|_{*}$ are not equivalent on $l^{1}(S)$.

Definition 3.5 (Picardello [22]). A subset $S$ of $G$ is called a weak Sidon set if given any $f \in l^{\infty}(G)$ there exists $u \in B(G)$ such that $f(x)=u(x)$ for $x \in S$.

Using standard results in functional analysis, one sees that a set $S$ in $G$ is weak Sidon if and only if $\|\cdot\|_{1}$ and $\|\cdot\|_{*}$ are equivalent on $l^{\prime}(S)$. Therefore, the above proposition states that if $S$ contains large squares then it is not a weak Sidon set. When $G$ is abelian, a weak Sidon set is just a Sidon set in the usual sense. Note that [22] also contains the definitions of Sidon sets and strong Sidon sets for general discrete groups. 
Lemma 3.6. Suppose that $S$ is a subset of $G$ and that there exist $x_{1}, \ldots, x_{n}$ in $G$ such that $x_{1} S \cup x_{2} S \cup \cdots \cup x_{n} S$ contains large squares. Then $S$ also contains large squares.

Proof. Note that

$$
x_{1} S \cup \cdots \cup x_{n} S \subset\left(x_{2} S \cup \cdots \cup x_{n} S\right) \cup x_{1} x_{2}^{-1}\left(x_{2} S \cup \cdots \cup x_{n} S\right) .
$$

Therefore it suffices to prove this lemma for the case $n=2$.

Assume that $S \cup x S$ contains large squares and let $k \in \mathbf{N}$ be given. Then there exist $A, B \subset G$ such that $A \cdot B \subset S \cup x S,|A|=2 k \cdot\left(\begin{array}{c}2 k \\ k\end{array}\right),|B|=2 k$ and $|A \cdot B|=$ $4 k^{2}\left(\begin{array}{c}2 k \\ k\end{array}\right)$. (As usual $\left(\begin{array}{c}2 k \\ k\end{array}\right)=(2 k) ! /(k !)^{2}$.) For each $a \in A$, let $C(a)=\{b \in B: a b \in S\}$ and $D(a)=\{b \in B: a b \in x S\}$. Since $C(a) \cup D(a)=B$, either $|C(a)| \geqslant k$ or $|D(a)| \geqslant k$. Therefore, either (i) there exists $A_{1} \subset A$ such that $\left|A_{1}\right|=k \cdot\left(\begin{array}{c}2 k \\ k\end{array}\right)$ and if $a \in A_{1}$ then $|C(a)| \geqslant k$ or (ii) there exists $A_{2} \subset A$ such that $\left|A_{2}\right|=k \cdot\left(\begin{array}{c}2 k \\ k\end{array}\right)$ and if $a \in A_{2},|D(a)| \geqslant k$.

Suppose that (i) holds. For each $a \in A_{1}$, choose $C_{1}(a) \subset C(a)$ such that $\left|C_{1}(a)\right|$ $=k$. Let $\mathscr{F}=\{F: F \subset B,|F|=k\}$. Note that $|\mathscr{F}|=\left(\begin{array}{c}2 k \\ k\end{array}\right)$. If $F \in \mathscr{F}$, set $\alpha(F)=\{a$ $\left.\in A_{1}: C_{1}(a)=F\right\}$. Then $\cup\{\alpha(F): F \in \mathscr{F}\}=A_{1}$. Hence

$$
\left|A_{1}\right|=k \cdot\left(\begin{array}{c}
2 k \\
k
\end{array}\right)=\sum\{|\alpha(F)|: F \in \mathscr{F}\} .
$$

Since $|\mathscr{F}|=\left(\begin{array}{c}2 k \\ k\end{array}\right)$, there exists at least one $F_{0} \in \mathscr{F}$ with $\left|\alpha\left(F_{0}\right)\right| \geqslant k$. Choose $E \subset \alpha\left(F_{0}\right)$ such that $|E|=k$. Then $E \cdot F_{0}$ is a $k$-square contained in $S$.

If (ii) holds then there exists a $k$-square $E \cdot F$ contained in $x S$. Then $\left(x^{-1} E\right) \cdot F$ is a $k$-square contained in $S$. This completes the proof.

REMARK. By symmetry, if there exist $y_{1}, \ldots, y_{m}$ such that $S y_{1} \cup \cdots \cup S y_{m}$ contains large squares then $S$ also contains large squares. Moreover, if $X$ and $Y$ are finite subsets of $G$ such that $X \cdot S \cdot Y$ contains large squares then $X \cdot S$ contains large squares and hence $S$ contains large squares.

Definition 3.7. A subset $S$ of $G$ is said to be relatively dense if there exist two finite subsets $X$ and $Y$ of $G$ such that $G=X \cdot S \cdot Y$.

REMARKS. (1) When $G=\mathbf{Z}$, the additive group of integers, the above definition of course coincides with $\mathrm{H}$. Bohr's definition of relative density, see [3].

(2) Suppose that $S$ is a relatively dense subset of $G$, say, $G=X \cdot S \cdot Y$, as in the above definition, and $F$ is a finite subset of $S$. Choose $s_{0} \in S \backslash F$. Then $G=(X \cup X$ $\left.\cdot F \cdot Y s_{0}^{-1}\right) \cdot(S \backslash F) \cdot(Y \cup\{e\})$. Therefore, $S \backslash F$ is also relatively dense.

Since $G$ itself clearly contains large squares, by the remark prior to Definition 3.7, we see that a relatively dense set contains large squares. Combining this observation with Proposition 3.4, we get

COROLlaRY 3.8. If $S$ is a relatively dense subset of $G$ then it contains large squares and therefore it is not a weak Sidon set.

LEMMA 3.9. Let $S$ be a relatively dense subset of $G$ and let $F$ be a finite subset of $G$, $e \notin F$. Then there exists a relatively dense set $E$ of $G$ such that $E \subset S$ and $(x E \cap E)$ $\cup(E x \cup E)$ is empty if $x \in F$. 
Proof. Let $\mathcal{P}$ be the family of all subsets $P$ of $S$ such that

$$
(x P \cap P) \cup(P x \cap P)=\varnothing, \quad \text { if } x \in F .
$$

By the Zorn's lemma, $\mathcal{P}$ has a maximal element $E$. We claim that

$$
S \subset F_{1} \cdot E \cdot F_{1}
$$

where $F_{1}=F \cup F^{-1} \cup\{e\}$. Indeed if (3.3) were not true then one could choose $s \in S \backslash\left(F_{1} \cdot E \cdot F_{1}\right)$. It is not hard to check that $E \cup\{s\}$ would then be a member of $\mathcal{P}$. Since $s \notin E$, this would contradict the maximality of $E$ in $\mathscr{P}$. Therefore (3.3) holds true. Since $S$ is relatively dense in $G$ there exist finite subsets $X$ and $Y$ of $G$ such that $G=X \cdot S \cdot Y$. By (3.3), $G=\left(X \cdot F_{1}\right) \cdot E \cdot\left(F_{1} \cdot Y\right)$. Therefore $E$ is relatively dense.

Proposition 3.10. Let $G$ be an infinite discrete group. Then there exist a sequence of mutually disjoint $n$-squares $C_{n}, n=1,2, \ldots$, such that $T=\cup_{n=1}^{\infty} C_{n}$ is a t-set. (By Proposition 3.4, $T$ is not a weak Sidon set.)

Proof. Clearly, we only have to consider the case that $G$ is countably infinite. Write $G \backslash\{e\}=F_{1} \cup F_{1} \cup \cdots$ where $F_{1} \subset F_{2} \subset \cdots$ and each $F_{n}$ is a finite set. Applying the above lemma inductively, we can obtain a sequence of relatively dense sets $S_{n}$ of $G, n=1,2, \ldots$, such that

$$
S_{1} \supset S_{2} \supset \cdots,
$$

and

$$
\left(x S_{n} \cap S_{n}\right) \cup\left(S_{n} x \cap S_{n}\right)=\varnothing, \quad \text { if } x \in F_{n} .
$$

Suppose that we have chosen $i$-squares, $C_{i}, i=1,2, \ldots, n$, such that $C_{i} \subset S_{i}$, $i=1,2, \ldots, n$ and $C_{i} \cap C_{j}=\varnothing$ if $1 \leqslant i, j \leqslant n, i \neq j$. Since $S_{n+1}$ is relatively dense and $C_{1} \cup \cdots \cup C_{n}$ is finite, as we have noted earlier, $S_{n+1} \backslash\left(C_{1} \cup \cdots \cup C_{n}\right)$ is again relatively dense. Therefore, there exists an $(n+1)$-square

$$
C_{n+1} \subset S_{n+1} \backslash\left(C_{1} \cup \cdots \cup C_{n}\right) \text {. }
$$

Thus, by induction, we have a sequence of $i$-squares $\left\{C_{i}\right\}$ such that

$$
\begin{aligned}
C_{i} \subset S_{i}, & i=1,2, \ldots, \\
C_{i} \cap C_{j}=\varnothing, & \text { if } i \neq j .
\end{aligned}
$$

Let $T=\cup_{i=1}^{\infty} C_{i}$. We claim that $T$ is a $t$-set. Let $x \in G, x \neq e$. Then $x \in F_{n}$ for some $n$. Write $T=T_{1} \cup T_{2}$ where $T_{1}=C_{1} \cup C_{2} \cup \cdots \cup C_{n-1}$ and $T_{2}=C_{n} \cup C_{n+1}$ $\cup \cdots$. Then

$$
x T \cap T=\left(x T_{1} \cap T_{1}\right) \cup\left(x T_{1} \cap T_{2}\right) \cup\left(x T_{2} \cap T_{1}\right) \cup\left(x T_{2} \cap T_{2}\right) .
$$

Now by (3.6) and (3.4),

$$
T_{2}=C_{n} \cup C_{n+1} \cup \cdots \subset S_{n} \cup S_{n+1} \cup \cdots=S_{n} .
$$

Since $x \in F_{n}$, by (3.5), $x T_{2} \cap T_{2} \subset x S_{n} \cap S_{n}=\varnothing$. Therefore,

$$
|x T \cap T| \leqslant 3\left|T_{1}\right|<\infty \text {. }
$$

Similarly, $|T x \cap T| \leqslant 3\left|T_{1}\right|$. Thus $T$ is a $t$-set, as claimed. 
LemMa 3.11. Suppose that $S$ is a non(weak Sidon) subset of $G$. Then there exists $f \in l^{\infty}(G)$ such that suppt $f \subset S,\|f\|_{\infty}=1$ and $\|f-u\|_{S} \geqslant 1$ whenever $u \in B(G)$. (Here for a bounded function $g$ on $G,\|g\|_{S}=\sup \{|g(x)|: x \in S\}$.)

Proof. Take any $x_{1} \in S$ and let $h_{1}=\delta_{x_{1}}$. Suppose that we have constructed $h_{i} \in l^{\prime}(S)$ with mutually disjoint finite supports $K_{i}, i=1,2, \ldots, n$, such that $\left\|h_{n}\right\|_{1}$ $=1$ and $\left\|h_{n}\right\|_{*} \leqslant 1 / n$. Then $S \backslash\left(K_{1} \cup \cdots \cup K_{n}\right)$ is again a nonweak Sidon set. So there exists $h_{n+1} \in l^{\prime}\left(S \backslash\left(K_{1} \cup \cdots \cup K_{n}\right)\right)$ such that suppt $h_{n+1}=K_{n+1}$ is finite, $\left\|h_{n+1}\right\|_{1}=1$ and $\left\|h_{n+1}\right\|_{*} \leqslant 1 /(n+1)$. By induction we have a sequence of functions $\left\{h_{n}\right\}$ in $l^{1}(S)$ with finite supports $K_{n}$ such that

$$
\begin{aligned}
K_{i} \cap K_{j} & =\varnothing \quad \text { if } i \neq j, \\
\left\|h_{n}\right\|_{1} & =1, \quad\left\|h_{n}\right\|_{*} \leqslant 1 / n, \quad n=1,2, \ldots
\end{aligned}
$$

Let $f_{n}(x)=\left|h_{n}(x)\right| / h_{n}(x)$ if $x \in K_{n}$ and $=0$ otherwise. Then $\Sigma_{x \in G} f_{n}(x) h_{n}(x)=1$. Let $f(x)=\sum_{n=1}^{\infty} f_{n}(x)$. Then $f \in l^{\infty}(G),\|f\|_{\infty}=1$ and suppt $f=\cup_{n=1}^{\infty} K_{n} \subset S$.

Let $u \in B(G)$. Then $\left|\left\langle u, h_{n}\right\rangle\right| \leqslant\|u\|\left\|h_{n}\right\|_{*} \leqslant\|u\| / n$. So, for each $n$,

$$
\begin{aligned}
1 & =\sum_{x \in G} f(x) h_{n}(x) \\
& =\left|\sum_{x \in G}(f(x)-u(x)) h_{n}(x)+\sum_{x \in G} u(x) h_{n}(x)\right| \\
& \leqslant\|f-u\|_{S}\left\|h_{n}\right\|_{1}+\left|\left\langle u, h_{n}\right\rangle\right| \\
& \leqslant\|f-u\|_{S}+\|u\| / n .
\end{aligned}
$$

Since $n$ can be arbitrarily large, $\|f-u\|_{S} \geqslant 1$.

THEOREM 3.12. Suppose that $G$ is an infinite discrete group. Then $B(G)^{-} \subset W(G)$. In fact, the quotient Banach space $W(G) / B(G)^{-}$contains a linear isometric copy of $l^{\infty}$.

Proof. By Proposition 3.10, there exists a sequence of mutually disjoint $n$-squares $C_{n}, n=1,2, \ldots$, such that $T=\cup_{n=1}^{\infty} C_{n}$ is a $t$-set. Write $\mathbf{N}=\cup_{n=1}^{\infty} N_{n}$ where, for each $n, N_{n}$ is infinite and the $N_{n}$ 's are mutually disjoint. Let $T_{n}=\cup_{k \in N_{n}} C_{k}$. Then $T_{n}$ contains large squares and hence, by Proposition 3.4, is not a weak Sidon set. By Lemma 3.11 , there exists $f_{n} \in l^{\infty}(G)$ such that suppt $f_{n} \subset T_{n},\left\|f_{n}\right\|_{\infty}=1$ and $\left\|f_{n}-u\right\|_{T_{n}} \geqslant 1$ if $u \in B(G)$.

If $\left(c_{n}\right) \in l^{\infty}$ then $\sum_{n=1}^{\infty} c_{n} f_{n} \in l^{\infty}(G)$ and $\left\|\sum_{n=1}^{\infty} c_{n} f_{n}\right\|_{\infty}=\left\|\left(c_{n}\right)\right\|_{\infty}$. Since $\operatorname{suppt}\left(\sum_{n=1}^{\infty} c_{n} f_{n}\right) \subset \cup_{n=1}^{\infty} T_{n} \subset T$ and $T$ is a $t$-set, $\sum_{n=1}^{\infty} c_{n} f_{n} \in W(G)$. Let $\alpha: l^{\infty} \rightarrow$ $W(G) / B(G)^{-}$be defined by $\alpha\left(\left(c_{n}\right)\right)=\sum_{n=1}^{\infty} c_{n} f_{n}+B(G)^{-}$. Then, for $k \in \mathbf{N}$,

$$
\begin{aligned}
\left\|\alpha\left(\left(c_{n}\right)\right)\right\| & =\inf \left\{\left\|\sum_{n=1}^{\infty} c_{n} f_{n}+u\right\|_{\infty}: u \in B(G)\right\} \\
& \geqslant \inf \left\{\left\|\sum_{n=1}^{\infty} c_{n} f_{n}+u\right\|_{T_{k}}: u \in B(G)\right\} \\
& =\left|c_{k}\right| \inf \left\{\left\|f_{k}+u\right\|_{T_{k}}: u \in B(G)\right\} \\
& \geqslant\left|c_{k}\right| .
\end{aligned}
$$


So $\left\|\alpha\left(\left(c_{n}\right)\right)\right\| \geqslant\left\|\left(c_{n}\right)\right\|_{\infty}$. On the other hand, $\left\|\alpha\left(\left(c_{n}\right)\right)\right\| \leqslant\left\|\sum_{n=1}^{\infty} c_{n} f_{n}\right\|_{\infty}=\left\|\left(c_{n}\right)\right\|_{\infty}$. Therefore, $\left\|\alpha\left(\left(c_{n}\right)\right)\right\|=\left\|\left(c_{n}\right)\right\|_{\infty}$, i.e., $\alpha$ is an isometry.

REMARKS. (1) If $S$ is a nonweak Sidon set in $G$, then there exists a sequence $\left\{S_{n}\right\}_{n=1}^{\infty}$ of mutally disjoint nonweak Sidon subsets of $S$. Indeed, construct $\left\{K_{i}\right\}$ as in the proof of Lemma 3.11. Write $\mathbf{N}=\cup_{n=1}^{\infty} N_{n}$ such that each $N_{n}$ is infinite and the $N_{n}$ 's are mutually disjoint. Let $S_{n}=\cup_{i \in N_{n}} K_{i}$. Then $\left\{S_{n}\right\}$ is a mutually disjoint sequence of nonweak Sidon subsets of $S$.

(2) If $G$ is an infinite discrete group then it contains a subset $S$ such that $\chi_{s} \in W(G) \backslash B(G)^{-}$. Indeed, let $t$ be a $t$-set such that it is not weak Sidon. Then there exists $f \in l^{\infty}(G)$, suppt $f \subset T$ and $\|f-u\|_{T} \geqslant 1$ if $u \in B(G)$. Choose a simple function $g=\sum_{k=1}^{u} c_{k} \chi_{S_{k}}$ such that $S_{k} \subset T$ and $\|f-g\|_{\infty}<\frac{1}{2}$. Then $g \notin B(G)^{-}$and hence for some $k, \chi_{s_{k}} \notin B(G)^{-}$. Since $S_{k}$ is contained in $T$, it is a $t$-set, and hence $\chi_{s_{k}} \in W(G)$.

4. [IN]-groups and nilpotent groups. In this section $G$ will again denote a general noncompact locally group.

Lemma 4.1. Let $H$ be an open subgroup of $G$. Extend $f \in C(H)$ to $f^{\prime} \in C(G)$ by setting $f^{\prime}(x)=0$ if $x \in G \backslash H$.

(a) If $f \in W(H)$ then $f^{\prime} \in W(G)$.

(b) If $f \in B(H)$ then $f^{\prime} \in B(G)$.

Proof. For (a), see [5, Lemma 2.4]. For (b), see Hewitt and Ross [19, p. 280].

LEMMA 4.2. Let $H$ be a closed normal subgroup of $G, \theta$ the natural homomorphism of $G$ onto $G / H$.

(a) If $f \in W(G / H)$ then $f \circ \theta \in W(G)$.

(b) If $f \in B(G / H)$ then $f \circ \theta \in B(G)$.

Proof. For (a), see [4, Theorem 1.8]. For (b), see [14, p. 202].

If $H$ is a closed normal subgroup of $G$, and $f \in W(G)$, set

$$
f^{H}(\dot{x})=m_{H}\left(f_{x}\right), \quad \dot{x}=x H \in G / H,
$$

where $f_{x} \in W(H)$ is defined by $f_{x}(t)=f(x t), t \in H$. Note that since $m_{H}$ is $H$ invariant, $f^{H}$ is well defined.

LEMMA 4.3. Let $H$ be a closed normal subgroup of $G$.

(a) If $f \in W(G)$ then $f^{H} \in W(G / H),\left\|f^{H}\right\|_{\infty} \leqslant\|f\|_{\infty}$ and $(g \circ \theta)^{H}=g$ if $g \in$ $W(G / H)$.

(b) If $f \in B(G)$ then $f^{H} \in B(G / H)$.

Proof. For (a), see [7, Lemma 2.3]. The proof of (b) is contained in the proof of the Theorem in [6].

For convenience, a sequence of functions $\left\{f_{n}\right\}$ in $C(G)$ will be called a (*)-sequence if $\left\|f_{n}\right\|_{\infty}=1$, the supports of the $f_{n}$ 's are mutually disjoint, for each sequence $\left(c_{n}\right) \in l^{\infty}, \Sigma_{n=1}^{\infty} c_{n} f_{n} \in W(G)$ and

$$
\left\|\sum_{n=1}^{\infty} c_{n} f_{n}-u\right\|_{\infty} \geqslant\left\|\left(c_{n}\right)\right\|_{\infty}
$$


for each $u \in B(G)$. Note that if $G$ has a (*)-sequence $\left\{f_{n}\right\}$ then $\left(c_{n}\right) \mapsto \sum_{n=1}^{\infty} c_{n} f_{n}+$ $B(G)^{-}$is a linear isometry of $l^{\infty}$ into the quotient Banach space $W(G) / B(G)^{-}$. Therefore to show that $W(G) / B(G)^{-}$contains a linear isometry copy of $l^{\infty}$ one only has to exhibit a (*)-sequence of $G$.

LEMMA 4.4. (a) Suppose that $H$ is an open subgroup of $G$ and $H$ has $a(*)$-sequence. Then $G$ also has a (*)-sequence.

(b) Suppose $H$ is a closed normal subgroup of $G$ and $G / H$ has $a(*)$-sequence. Then $G$ has a (*)-sequence.

Proof. (a) If $\left\{f_{n}\right\}$ is a (*)-sequence of $H$, let $f_{n}^{\prime}(x)=f_{n}(x)$ if $x \in H$ and $f_{n}^{\prime}(x)=0$ if $x \in G \backslash H$. Then, applying Lemma 4.1 , it is easy to see that $\left\{f_{n}^{\prime}\right\}$ is a (*)-sequence of $G$.

(b) We will keep the notations in Lemmas 4.2 and 4.3. Assume that $\left\{f_{n}\right\}$ is a (*)-sequence of $G / H$. We claim that $\left\{f_{n} \circ \theta\right\}$ is a $(*)$-sequence of $G$. Clearly $\left\|f_{n} \circ \theta\right\|_{\infty}=1$ and the supports of the $f_{n} \circ \theta$ 's are mutually disjoint. Let $\left(c_{n}\right) \in l^{\infty}$. Then, by Lemma 4.2(a), $\sum_{n=1}^{\infty} c_{n}\left(f_{n} \circ \theta\right)=\left(\sum_{n=1}^{\infty} c_{n} f_{n}\right) \circ \theta \in W(G)$ and it is easy to see that $\left(\sum_{n=1}^{\infty} c_{n}\left(f_{n} \circ \theta\right)\right)^{H}=\sum_{n=1}^{\infty} c_{n} f_{n}$. For $u \in B(G)$,

$$
\begin{aligned}
\left\|\sum_{n=1}^{\infty} c_{n}\left(f_{n} \circ \theta\right)-u\right\|_{\infty} & \geqslant\left\|\left(\sum_{n=1}^{\infty} c_{n}\left(f_{n} \circ \theta\right)-u\right)^{H}\right\|_{\infty} \\
& =\left\|\sum_{n=1}^{\infty} c_{n} f_{n}-u^{H}\right\|_{\infty} \geqslant\left\|\left(c_{n}\right)\right\|_{\infty},
\end{aligned}
$$

since, by Lemma 4.3(b), $u^{H} \in B(G / H)$ and $\left\{f_{n}\right\}$ is a (*)-sequence of $G / H$. Thus, $\left\{f_{n} \circ \theta\right\}$ is a $(*)$-sequence of $G$, as claimed.

In the proof of Theorem 3.12 we show that infinite discrete groups have (*)-sequences. We want to show that $\mathbf{R}$, the additive group of real numbers also has a (*)-sequence. Indeed let $\left\{S_{n}\right\}$ be a sequence of mutually disjoint subsets of $\mathbf{Z}$ such that each $S_{n}$ is non-Sidon and $\cup_{n=1}^{\infty} S_{n}$ is a $t$-set of $\mathbf{Z}$. Choose $g_{n} \in l^{\infty}(Z)$ such that $\left\|g_{n}\right\|_{\infty}=1$, suppt $g_{n} \subset S_{n}$ and $\left\|g_{n}-u\right\|_{S_{n}} \geqslant 1$ if $u \in B(\mathbf{Z})$. Extend $g_{n}$ to a bounded continuous function $f_{n}$ on $\mathbf{R}$ as follows: $f_{n}(x)=g_{n}(k)(1-8|x-k|)$, if $|x-k| \leqslant \frac{1}{8}$ for some $k \in S_{n}$ and $f_{n}(x)=0$ otherwise. Since $\cup_{n=1}^{\infty} S_{n}$ is a $t$-set of $\mathbf{Z}$, by Rudin [24, Lemma 3] or Dunkl-Ramirez [11, p. 45], if $\left(c_{n}\right) \in l^{\infty}, \sum_{n=1}^{\infty} c_{n} f_{n} \in W(\mathbf{R})$. Clearly, the supports of the $f_{n}$ 's are mutually disjoint and $\left\|f_{n}\right\|_{\infty}=1$. Furthermore, for $u \in B(\mathbf{R})$ and $\left(c_{n}\right) \in l^{\infty}$,

$$
\left\|\sum_{n=1}^{\infty} c_{n} f_{n}-u\right\|_{\infty} \geqslant\left\|\sum_{n=1}^{\infty} c_{n} f_{n}-u\right\|_{\mathbf{Z}}=\left\|\sum_{n=1}^{\infty} c_{n} g_{n}-\left.u\right|_{\mathbf{Z}}\right\|_{\mathbf{Z}} \geqslant\left\|\left(c_{n}\right)\right\|_{\infty},
$$

since $\left\{g_{n}\right\}$ is a (*)-sequence of $\mathbf{Z}$. So $\left\{f_{n}\right\}$ is a $(*)$-sequence of $\mathbf{R}$.

We are ready now for the main results.

THEOREM 4.5. Let $G$ be a noncompact $[I N]$-group. Then $G$ is not an Eberlein group. Indeed, $W(G) / B(G)^{-}$contains a linear isometric copy of $l^{\infty}$. 
Proof. It suffices to construct a (*)-sequence for $G$. Since $G$ is an $[I N]$-group, by Grosser-Moskowitz' structure theorem [17, Theorem 2.13] there exists an open normal subgroup $H$ of $G$ such that $H$ has a compact normal subgroup $K$ such that $H / K$ is a vector group.

If $G / H$ is an infinite (discrete) group then it has a (*)-sequence and hence, by Lemma 4.4(b), $G$ also has a (*)-sequence.

If $G / H$ is finite then $H$ is noncompact and hence the vector group $H / K$ is noncompact. Therefore, $\mathbf{R}$ is a continuous homomorphic image of $H$. Since $\mathbf{R}$ has a (*)-sequence, by Lemma 4.4(b), $H$ also has a (*)-sequence. Since $H$ is open in $G$, by Lemma 4.4(a), $G$ has a (*)-sequence. This completes the proof of the theorem.

If $G$ is a locally compact group and $Z(G)$ is its center, then $G$ is called a [ $Z$ ]-group if $G / Z(G)$ is compact, cf. [16]. It is known that [Z]-groups are [SIN]-groups, cf. [16]. Recall that a locally compact group $G$ is called a [SIN]-group if the left and right uniform structures of $g$ are equivalent, cf. [17]. In particular, [SIN]-groups are [IN]-groups.

THEOREM 4.6. If $G$ is a noncompact locally compact nilpotent group then $G$ is not an Eberlein group. Indeed, $W(G) / B(G)^{-}$contains a linear isometric copy of $l^{\infty}$.

Proof. We will prove that $G$ has a $(*)$-sequence. Since $G$ is nilpotent, we can consider its upper central sequence

$$
G=G_{0} \supset G_{1} \supset \cdots \supset G_{n}=(e)
$$

where each $G_{i}$ is a closed normal subgroup of $G$ and $G_{i-1} / G_{i}$ is the center of $G / G_{i}$, $i=1,2, \ldots, n$; in particular, $G_{n-1}$ is the center of $G$. We shall call $n$ the rank of $G$.

If $n=1$, then $G$ is abelian. By Theorem 4.5, $G$ has a $(*)$-sequence. Assume that every nilpotent group with rank $\leqslant n-1$ has a $(*)$-sequence. Let $G$ be a nilpotent group of rank $n$. If $G / G_{n-1}$ is noncompact then, being a noncompact nilpotent group of rank $n-1$, it has a $(*)$-sequence. By Lemma 4.4(b), $G$ also has a (*)-sequence. If $G / G_{n-1}$ is compact then $G$ is a [Z]-group and hence an [IN]-group. By Theorem 4.5, $G$ has a (*)-sequence.

REMARK. If the center $Z(G)$ of a locally compact group $G$ is noncompact then $B(G)^{-} \subset W(G)$. Indeed, choose $f \in W(Z(G)) \backslash B(Z(G))^{-}$. By Theorem 1 of Cowling and Rodway [8], there exists $F \in W(G)$ such that $F \mid Z(G)=f$. Clearly $F \notin B(G)^{-}$.

5. W.a.p. functions on the direct products of groups. $W(G)$ being a commutative $C^{*}$-algebra is *-isomorphic to $C\left(G^{\omega}\right)$ where $G^{\omega}$ is the maximal ideal space of $W(G)$. Since $W(G)$ separates the points of $G$, we may consider $G$ as a subset of $G^{\omega}$ and $W(G)$ is exactly the space of those continuous functions on $G$ which can be extended to continuous functions on $G^{\omega}$. $G^{\omega}$ is called the w.a.p. compactification of $G$. It has a natural semigroup structure and its multiplication is separately continuous. This aspect of w.a.p. functions was first studied in detail by de Leeuw and Glicksberg in [9].

If $G$ and $H$ are locally compact groups, we will denote their direct product by $G \times H$. For $f \in C(G), g \in C(H), f \otimes g \in C(G \times H)$ is defined by $(f \otimes g)(x, y)=$ $f(x) g(y),(x, y) \in G \times H . W(G) \otimes W(H)$, the linear span of functions of the form 
$f \otimes g, f \in W(G), g \in W(H)$, is a linear subalgebra of $W(G \times H)$. Since $W(G) \approx$ $C\left(G^{\omega}\right)$ and $W(H) \approx C\left(H^{\omega}\right)$, by the Stone-Weierstrass theorem, $(W(G) \otimes W(H))^{-}$ $\approx C\left(G^{\omega} \times H^{\omega}\right)$. Therefore, as noted in Milnes [21, Theorem 1.2], for $f \in W(G \times H)$ the following conditions are equivalent: (i) $f \in(W(G) \otimes W(H))^{-}$, (ii) $f$ can be extended to a continuous function on $G^{\omega} \times H^{\omega}$ and (iii) $A_{f}=\left\{f_{x}: x \in G\right\}$ is a relatively compact subset of $W(H)$ (with respect to sup norm topology) where $f_{x} \in W(H)$ is defined by $f_{x}(y)=f(x, y), y \in H$. In particular, $(G \times H)^{\omega}=G^{\omega} \times$ $H^{\omega}$ if and only if $(W(G) \otimes W(H))^{-}=W(G \times H)$. It is pointed out in a footnote in de Leeuw and Glicksberg [10, p. 105] that for abelian groups, in general, $(G \times H)^{\omega}$ $\neq G^{\omega} \times H^{\omega}$. See also [2, p. 171 and 21, Theorem 1.3]. We will prove that for a large class of groups (including all noncompact abelian groups) $(G \times H)^{\omega} \neq G^{\omega} \times H^{\omega}$.

As in [5], a locally compact group $G$ is said to have property (E) if $G$ contains a subset $X$ such that $X$ is not relatively compact and for each neighborhood $U$ of $e$, the set $\cap\left\{x^{-1} U x: x \in X \cup X^{-1}\right\}$ is again a neighborhood of $e$. Such a set $X$ will be called an (E)-set of $G$. For example, noncompact [SIN]-groups and groups with noncompact centers have property (E).

THEOREM 5.1. Suppose that $G$ and $H$ are noncompact locally compact groups with property (E). Then $(W(G) \otimes W(H))^{-} \subset W(G \times H)$; in other words, $(G \times H)^{\omega} \neq G^{\omega}$ $\times H^{\omega}$.

Proof. We will exhibit a function $f \in W(G \times H)$ such that $A_{f}=\left\{f_{x}: x \in G\right\}$ is not relatively compact in $W(H)$. Choose nonrelatively compact symmetric $E$-sets $A$ and $B$ in $G$ and $H$ respectively. Fix symmetric compact neighborhoods $U$ of $e_{G}$ and $V$ of $e_{H}$. Then there exist compact symmetric neighborhoods $U_{1}$ of $e_{G}$ and $V_{1}$ of $e_{H}$ such that $a^{-1} U_{1} a \subset U$ if $a \in A$ and $b^{-1} V_{1} b \subset V$ if $b \in B$. Select $a_{n} \in A, b_{n} \in B$ inductively such that for $n \geqslant 2$,

$$
\begin{aligned}
& a_{n} \notin\left\{\prod_{j=1}^{7} x_{j}: x_{j} \in U_{1} \cup\left\{a_{1}, \ldots, a_{n-1} ; a_{1}^{-1}, \ldots, a_{n-1}^{-1}\right\}\right\}, \\
& b_{n} \notin\left\{\prod_{j=1}^{7} y_{j}: y_{j} \in V_{1} \cup\left\{b_{1}, \ldots, b_{n-1} ; b_{1}^{-1}, \ldots, b_{n-1}^{-1}\right\}\right\} .
\end{aligned}
$$

Choose $g \in C(G)$ and $h \in C(H)$ such that $g\left(e_{G}\right)=h\left(e_{H}\right)=1,0 \leqslant g \leqslant 1,0 \leqslant h \leqslant$ 1 , and suppt $g \subset U_{1}$, suppt $h \subset V_{1}$. Let

$$
f(x, y)=\sum_{n=1}^{\infty} g\left(a_{n}^{-1} x\right) h\left(b_{n}^{-1} y\right) .
$$

Then $f \in W(G \times H)$; see the proof of $[5$, Theorem 4.6] for details. Note that

$$
\begin{aligned}
f_{a_{k}}(y) & =f\left(a_{k}, y\right)=\sum_{n=1}^{\infty} g\left(a_{n}^{-1} a_{k}\right) h\left(b_{n}^{-1} y\right) \\
& =h\left(b_{k}^{-1} y\right), \quad \text { since } a_{n} U_{1} \cap a_{k} U_{1}=\varnothing \text { if } n \neq k, \\
& =\left(l_{b_{k}} h\right)(y), \quad y \in H .
\end{aligned}
$$


Since suppt $\left(l_{b_{k}} h\right) \subset b_{k} V_{1}$ and $b_{k} V_{1} \cap b_{n} V_{1}=\varnothing$ if $k \neq n$, we see that $\left\{f_{a_{k}}\right\}_{k \geqslant 1}=$ $\left\{l_{b_{k}} h\right\}$ is not relatively compact in $C(H)$. Therefore $f \notin(W(G) \otimes W(H))^{-}$. This completes the proof.

LeMma 5.2. If $G_{1}$ and $H_{1}$ are continuous homomorphic images of $G$ and $H$ respectively and $\left(W\left(G_{1}\right) \otimes W\left(H_{1}\right)\right)^{-} \underset{\neq}{\subset} W\left(G_{1} \times H_{1}\right)$ then $(W(G) \otimes W(H))^{-} \underset{\neq}{\subsetneq}$ $W(G \times H)$.

Proof. Easy.

COROLlary 5.3. Let $G$ and $H$ be two noncompact locally compact groups. Then $W(G \times H) \supsetneqq(W(G) \otimes W(H))^{-}$if either (i) $G$ and $H$ are [IN]-groups or (ii) $G$ and $H$ are nilpotent groups.

Proof. If $G$ and $H$ are [ $I N$ ]-groups then there exist compact normal subgroups $K_{1}$ and $K_{2}$ of $G$ and $H$ respectively such that $G / K_{1}, H / K_{2}$ are (noncompact) [SIN]groups cf. [17, Theorem 2.5]. By Theorem 5.1, $\left(W\left(G / K_{1}\right) \otimes W\left(H / K_{2}\right)\right)^{-} \subsetneq$ $W\left(G / K_{1} \times H / K_{2}\right)$ and hence, by Lemma 5.2, $(W(G) \otimes W(H))^{-} \underset{\neq}{\subset} W(G \times H)$.

The nilpotent case can be proved by using the inductive argument given in the proof of Theorem 4.6. We omit the details.

ReMARKs. (1) It is also easy to prove that if $G$ is a noncompact [ $I N]$-group and $H$ is a noncompact nilpotent group then $(G \times H)^{\omega} \neq G^{\omega} \times H^{\omega}$.

(2) $[I N]$-groups and nilpotent groups may fail to have property (E). Indeed, let $G=\mathbf{R} \times \mathbf{R} \times \mathbf{T}$ with product topology and with multiplication given by

$$
(x, y, \exp (i \theta))\left(x^{\prime}, y^{\prime}, \exp \left(i \theta^{\prime}\right)\right)=\left(x+x^{\prime}, y+y^{\prime}, \exp \left(i\left(\theta+\theta^{\prime}+x y^{\prime}\right)\right)\right) .
$$

Then $G$ is a nilpotent $[I N]$-group without property (E). See [5, p. 185] for details.

Veech proved in [26] that if $G$ is a noncompact simple analytic group with finite center then $W(G)=\mathbf{C} \oplus C_{0}(G)=$ space of continuous functions on $G$ which are convergent at infinity. (Also see [7, Theorem 4.1] for the special case that $G=$ $S L(2, \mathbf{R})$.) Therefore, $G$ is minimally w.a.p. and $G^{\omega}=G^{*}$, the one point compactification of $G$. Furthermore, he showed that if $G_{1}, \ldots, G_{k}$ are simple analytic groups with finite centers then $G^{\omega}=G_{1}^{*} \times G_{2}^{*} \times \cdots \times G_{k}^{*}$. (Here $G_{j}$ can be compact. If $G_{j}$ is compact then $G_{j}^{*}=G_{j}$.) Therefore, $W(G)=\left(W\left(G_{1}\right) \otimes \cdots \otimes W\left(G_{k}\right)\right)^{-}$.

It is well known that if $G$ is a semisimple analytic group with finite center then there is a finite extension $G_{0}$ of $G$ such that $G_{0}=G_{1} \times \cdots \times G_{k}$ where $G_{i}$ is a simple analytic group with finite center. Therefore, by Lemma 5.2, if $G$ and $H$ are semisimple analytic groups with finite centers then $W(G \times H)=(W(G) \otimes W(H))^{-}$.

If $G$ and $H$ are Eberlein groups and $W(G \times H)=(W(G) \otimes W(H))^{-}$, then

$$
\begin{aligned}
B(G \times H)^{-} & \subset W(G \times H)=(W(G) \otimes W(H))^{-} \\
& =(B(G) \otimes B(H))^{-} \subset B(G \times H)^{-} .
\end{aligned}
$$

Therefore, $W(G \times H)=B(G \times H)^{-}$, i.e. $G \times H$ is also an Eberlein group. In [7] we noted the following two facts: (1) If $K$ is a compact normal subgroup of $G$ then $G / K$ is minimally w.a.p. if and only if $G$ is minimally w.a.p. (2) If $G$ is an extension of a noncompact group by another noncompact group then $G$ is not minimally w.a.p. Using these facts, we may summarize consequences of Veech's results as follows. 
THEOREM 5.4. (a) Each noncompact semisimple analytic group $G$ with finite center is an Eberlein group. $G$ is minimally w.a.p. if and only if $G$ has exactly one noncompact simple constituent.

(b) If $G$ and $H$ are semisimple analytic groups with finite centers then $W(G \times H)=$ $(W(G) \otimes W(H))^{-}$, or equivalently, $(G \times H)^{\omega}=G^{\omega} \times H^{\omega}$.

Let $M(n)=S O(n) \times \mathbf{R}^{n}$ (semidirect product) be the Euclidean motion group of $\mathbf{R}^{n}$. In [7], we showed that $M(n)$ is minimally w.a.p. if $n \geqslant 2$. Milnes proved in [21] that $(M(2) \times M(2))^{\omega}=M(2)^{\omega} \times M(2)^{\omega}$. (Note that $M(2)$ is solvable but not nilpotent. Compare with Corollary 5.3.) Therefore, $M(2) \times M(2)$ is a connected solvable Eberlein group; but it is not minimally w.a.p.

LemMa 5.5. Assume that $G=G_{1} \times \cdots \times G_{k}$ where $G_{i}$ is topologically isomorphic to some $M\left(n_{i}\right), n_{i} \geqslant 2$. Let $f \in W_{0}(G)$. Then $f\left(x_{1}, \ldots, x_{k}\right) \rightarrow 0$ as $\left(x_{1}, \ldots, x_{k}\right) \rightarrow$ $(\infty, \ldots, \infty)$.

Proof. In [5] we showed that if $f \in W_{0}(M(2))$ then $f(x) \rightarrow 0$ as $x \rightarrow \infty$. Milnes proved in [21] that if $f \in W_{0}(M(2) \times M(2))$ then $f\left(x_{1}, x_{2}\right) \rightarrow 0$ as $\left(x_{1}, x_{2}\right) \rightarrow(\infty, \infty)$. His arguments can be extended to the general case stated in the lemma by using results contained in the proof of Theorem 3.3 of [7]. We omit the details.

REMARK. If $G_{1}, G_{2}, \ldots, G_{k}$ are locally compact groups consider the following condition.

$$
\begin{aligned}
& \text { If } f \in W_{0}\left(G_{1} \times \cdots \times G_{k}\right) \text { then } f\left(x_{1}, \ldots, x_{k}\right) \rightarrow 0 \\
& \text { as }\left(x_{1}, \ldots, x_{k}\right) \rightarrow(\infty, \ldots, \infty) .
\end{aligned}
$$

It is easy to see that if $G_{1} \times \cdots \times G_{k}$ satisfies $(5.1)$ and if $G_{i_{j}}, \ldots, G_{i_{j}}$ is a subcollection of $G_{1}, \ldots, G_{k}$ then $G_{i} \times \cdots \times G_{i_{j}}$ also satisfies (5.1). In particular, each $G_{i}$ is minimally w.a.p., $i=1,2, \ldots, k$.

Proposition 5.6. If $G=G_{1} \times \cdots \times G_{k}$ satisfies (5.1) then $W(G)=\left(W\left(G_{1}\right)\right.$ $\left.\otimes \ldots \otimes W\left(G_{k}\right)\right)^{-}$. In particular, $G$ is an Eberlein group.

Proof. As remarked earlier, we have to show that each $f \in W(G)$ can be extended to a continuous function on $G_{1}^{\omega} \times \cdots \times G_{k}^{\omega}$. It suffices to show that if, for each $i, x_{\alpha_{i}}$ is a net in $G_{i}$ such that $\lim _{\alpha_{i}} x_{\alpha_{i}}=\tau_{i} \in G_{i}^{\omega}, i=1,2, \ldots, k$, then $\lim _{\alpha} f\left(x_{\alpha_{1}}, \ldots, x_{\alpha_{k}}\right)$ exists where $\alpha=\left(\alpha_{1}, \ldots, \alpha_{k}\right)$. For notational convenience, we will assume that $\tau^{\prime}=\left(\tau_{1}, \ldots, \tau_{j}\right) \in G_{1} \times \cdots \times G_{j}$ and $\tau^{\prime \prime}=\left(\tau_{j+1}, \ldots, \tau_{k}\right) \in\left(G_{j+1}^{\omega} \backslash G_{j+1}\right)$ $\times \cdots \times\left(G_{k}^{\omega} \backslash G_{k}\right)$, for some $j \leqslant k$. We will write $\alpha$ as $\left(\alpha^{\prime}, \alpha^{\prime \prime}\right)$ where $\alpha^{\prime}=\left(\alpha_{1}, \ldots, \alpha_{j}\right)$, $\alpha^{\prime \prime}=\left(\alpha_{j+1}, \ldots, \alpha_{k}\right)$ and $x_{\alpha}=\left(x_{\alpha_{1}}, \ldots, x_{\alpha_{k}}\right)$ as $\left(x_{\alpha^{\prime}}, x_{\alpha^{\prime \prime}}\right)$ where $x_{\alpha^{\prime}}=\left(x_{\alpha_{1}}, \ldots, x_{\alpha_{j}}\right)$ and $x_{\alpha^{\prime \prime}}=\left(x_{\sigma_{j+1}}, \ldots, x_{\alpha_{k}}\right)$.

For a fixed point

$$
x^{\prime}=\left(x_{1}, \ldots, x_{j}\right) \in G_{1} \times \cdots \times G_{j},
$$

define $f_{x^{\prime}} \in W\left(G_{j+1} \times \cdots \times G_{k}\right)$ by setting $f_{x^{\prime}}\left(x^{\prime \prime}\right)=f\left(x^{\prime}, x^{\prime \prime}\right)$ where $x^{\prime \prime}=$ $\left(x_{j+1}, \ldots, x_{k}\right) \in G_{j+1} \times \cdots \times G_{k}$. Then $f_{x^{\prime}}$ can be decomposed as $f_{x^{\prime}}=g_{x^{\prime}}+h_{x^{\prime}}$ where $g_{x^{\prime}} \in A P\left(G_{j+1} \times \cdots \times G_{k}\right)$ and $h_{x^{\prime}} \in W_{0}\left(G_{j+1} \times \cdots \times G_{k}\right)$. It is known that if $H_{1}$ and $H_{2}$ are two locally compact groups then

$$
A P\left(H_{1} \times H_{2}\right)=\left(A P\left(H_{1}\right) \otimes A P\left(H_{2}\right)\right)^{-} \subset\left(W\left(H_{1}\right) \otimes W\left(H_{2}\right)\right)^{-},
$$


see Berglund and Milnes [2, Theorem 2.3] for a simple proof. Applying this fact to our situation here, we see that $g_{x^{\prime}} \in\left(W\left(G_{j+1}\right) \otimes \cdots \otimes W\left(G_{k}\right)\right)^{-}$and hence $\lim _{\alpha^{\prime \prime}} g_{x^{\prime}}\left(x_{\alpha^{\prime \prime}}\right)$ exists. Since $\lim _{\alpha^{\prime \prime}} x_{\alpha^{\prime \prime}}=\tau^{\prime \prime} \in\left(G_{j+1}^{\omega} \backslash G_{j+1}\right) \times \cdots \times\left(G_{k}^{\omega} \backslash G_{k}\right)$ and $G_{j+1} \times \cdots \times G_{k}$ satisfies (5.1), $\lim _{\alpha^{\prime \prime}} h_{x^{\prime}}\left(x_{\alpha^{\prime \prime}}\right)=0$. Therefore,

$$
\lim _{\alpha^{\prime \prime}} f_{x^{\prime}}\left(x_{\alpha^{\prime \prime}}\right) \text { exists. }
$$

Note that, since $f$ is uniformly continuous on $G$ (see [4]),

$$
\lim _{\alpha^{\prime}}\left\|f_{x_{\alpha^{\prime}}}-f_{\tau^{\prime}}\right\|_{\infty}=0
$$

By (5.2) and (5.3) it is not hard to see that $\lim _{\alpha} f\left(x_{\alpha}\right)$ exists, as desired.

THEOREM 5.7. Assume that $G=G_{1} \times \cdots \times G_{k}$ where each $G_{i}$ is topologically isomorphic to $M\left(n_{i}\right)$ for some $n_{i} \geqslant 2$. then $W(G)=\left(W\left(G_{1}\right) \otimes \cdots \otimes W\left(G_{k}\right)\right)^{-}$or equivalently, $G^{\omega}=G_{1}^{\omega} \times \cdots \times G_{k}^{\omega}$. In particular, $G$ is an Eberlein group.

Proof. The result is a direct consequence of Lemma 5.5 and Proposition 5.6.

REMARK. We do not yet know whether $G_{1} \times \cdots \times G_{k}$ always satisfies (5.1) if each $G_{i}$ is a minimally w.a.p. group. In particular, we wonder whether there exist minimally w.a.p. groups $G$ and $H$ such that $G \times H$ is not an Eberlein group.

6. The inclusive relation $B(G) \cap C_{0}(G) \subset B_{c}(G)$. Recall that for a noncompact locally compact group $G, B(G) \cap C_{0}(G)=B_{c}(G)$ if and only if $B_{c}(G) \subset C_{0}(G)$ if and only if $A P(G) \oplus C_{0}(G)=B(G)^{-}$, see Lemma 2.1. For an abelian $G$, if $\mu$ is a bounded regular Borel measure on the dual group $\hat{G}$ of $G$ and if $\mu_{d}, \mu_{c}$ are the discrete part and continuous part of $\mu$ respectively, then the Godement's decomposition of $\hat{\mu}$ is $\hat{\mu}_{d}+\hat{\mu}_{c}$, cf. Eberlein [13]. If $G$ is noncompact and abelian then $\hat{G}$ is nondiscrete and hence contains a Cantor set $C$ which is also a Helson set. Let $\mu$ be a nonzero continuous bounded Borel measure supported by $C$ then $\hat{\mu} \in B_{c}(G) \backslash C_{0}(G)$, cf. [4, p. 74]. In this section we will consider generalizations of this fact to nonabelian groups.

LEMMA 6.1. (1) Let $\varphi$ be a continuous homomorphism of $G$ onto $H$. If either (a) $B_{c}(H) \not \subset C_{0}(H)$ or (b) both $H$ and $\operatorname{ker} \varphi$ are noncompact then $B_{c}(G) \not \subset C_{0}(G)$.

(2) If $K$ is a compact normal subgroup of $G$ and $B_{c}(G / K) \subset C_{0}(G / K)$ then $B_{c}(G) \subset C_{0}(G)$.

Proof. (1) Let $g \in G(H)$. Then, by Lemma 4.2(b), $g \circ \varphi \in B(G)$ and, by [5, Lemma 4.1], $m_{G}(g \circ \varphi)=m_{H}(g)$. If (a) holds, choose any $g \in B_{c}(H) \backslash C_{0}(H)$. Then $g \circ \varphi \in B_{c}(G) \backslash C_{0}(G)$. If (b) holds, choose any nonzero $g \in B_{c}(H)$, then $g \circ \varphi \in B_{c}(G) \backslash C_{0}(G)$.

(2) If $B_{c}(G) \not \subset C_{0}(G)$, choose $f \in B_{c}(G) \backslash C_{0}(G)$. They by Lemma 4.3(b), $f^{K} \in$ $B(G / K)$. Since $m_{G / K}\left(\left|f^{K}\right|\right)=0$ (see [7, Lemma 2.3]), $f^{K} \in B_{c}(G / K)$. On the other hand, it is easy to check that $f^{K}$ does not vanish at infinity, cf. [7, Lemma 2.4]. Therefore, $B_{c}(G / K) \not \subset C_{0}(G / H)$. 
THEOREM 6.2. Let $G$ be a connected solvable noncompact locally compact group. Then the following three statements are equivalent.

(1) $G$ is minimally w.a.p.

(2) $B_{c}(G) \subset C_{0}(G)$.

(3) $G / K(G)$ is topolocally isomorphic to the motion group $M(2)$ where $K(G)$ is the largest compact normal subgroup of $G$.

Proof. (1) $\Leftrightarrow(3)$ is [7, Theorem 3.1]. Clearly, (1) $\Rightarrow(2)$. The proof of $(2) \Rightarrow(3)$ can be proceeded as the proof of $(1) \Rightarrow(3)$, by applying Lemma 6.1 and the fact that $B_{c}(G) \not \subset C_{0}(G)$ if $G$ is a noncompact abelian group. We omit the details.

Arguing as in the proof of [7, Theorem 3.2] we can also obtain the following.

THEOREM 6.3. If $G$ is a connected noncompact locally compact amenable group and $B_{c}(G) \subset C_{0}(G)$ then $G / K(G)$ is topologically isomorphic to the semidirect product of a compact subgroup $K$ of $S O(n)$ and $\mathbf{R}^{n}$ where $n \geqslant 2$ and $K$ acts on $\mathbf{R}^{n}$ naturally and irreducibly.

REMARK. It is natural to ask whether if $G=K \times \mathbf{R}^{n}$ (semidirect product) where $K$ is a compact subgroup of $S O(n)$ and the action of $K$ on $\mathbf{R}^{n}$ is natural and irreducible then $B_{c}(G) \subset C_{0}(G)$. It is known that for such $G$, the coefficient functions of all infinite dimensional irreducible unitary representations vanish at infinity, see Baggett and Taylor [1].

THEOREM 6.4. Let $G$ be a noncompact locally compact group. If either the center of $G$ is noncompact or if $G$ is nilpotent then $B_{c}(G) \not \subset C_{0}(G)$.

Proof. If $Z(G)$, the center of $G$, and $G / Z(G)$ are noncompact then, by Lemma $6.1, B_{c}(G) \not \subset C_{0}(G)$. If $Z(G)$ is noncompact but $G / Z(G)$ is compact then $G$ is a noncompact $[Z]$-group. By a structure theorem of Grosser and Moskowitz [16, p. 331], $G=V \times H$ (direct product) where $V$ is a vector group and $H$ contains a compact open normal subgroup $K$ such that $H / K$ is abelian. Either $V$ or $H / K$ is noncompact. Therefore, either $B_{c}(V) \not \subset C_{0}(V)$ or $B_{c}(H / K) \not \subset C_{0}(H / K)$. By Lemma 6.1, $B_{c}(G) \not \subset C_{0}(G)$.

If $G$ is nilpotent, then the proof can be proceeded as that of Theorem 4.6. We omit the details.

REMARK. We believe that if $G$ is an infinite discrete group then $B_{c}(G) \not \subset C_{0}(G)$. But we do not have a proof yet.

\section{REFERENCES}

1. L. Baggett and K. F. Taylor, Riemann-Lebesgue subsets of $\mathbf{R}^{n}$ and representations which vanish at infinity, J. Funct. Anal. 28 (1978), 168-181.

2. J. F. Berglund and P. Milnes, Algebras of functions on semitopological left groups, Trans. Amer. Math. Soc. 222 (1976), 157-178.

3. H. Bohr, Almost periodic functions, Chelsea, New York, 1947.

4. R. B. Burckel, Weakly almost periodic functions on semigroups, Gordon \& Breach, New York, 1970.

5. C. Chou, Weakly almost periodic functions and almost convergent functions on a group, Trans. Amer. Math. Soc. 206 (1975), 175-200.

6. __ Uniform closures of Fourier-Stieltjes algebras, Proc. Amer. Math. Soc. 77 (1979), 99-102.

7. __ Minimally weakly almost periodic groups, J. Funct. Anal. 36 (1980), 1-17. 
8. M. Cowling and P. Rodway, Restrictions of certain function spaces to closed subgroups of locally compact groups, Pacific J. Math. 80 (1979), 91-104.

9. K. de Leeuw and I. Glicksberg, Applications to almost periodic compactifications, Acta Math. 105 (1961), 63-97.

10. Almost periodic functions on semigroups, Acta Math. 105 (1961), 99-140.

11. C. F. Dunkl and D. E. Ramirez, Topics in harmonic analysis, Appleton-Century-Crofts, New York, 1971

12. W. F. Eberlein, Abstract ergodic theorems and weak almost periodic functions, Trans. Amer. Math. Soc. 67 (1949), 217-240.

13. A note on Fourier-Stieltjes transforms, Proc. Amer. Math. Soc. 6 (1955), 310-313.

14. P. Eymard, L'algèbre de Fourier d'un groupes localement compact, Bull. Soc. Math. France 92 (1964), 181-236.

15. R. Godement, Les functions de type positif et la théorie de groupes, Trans. Amer. Math. Soc. 65 (1948), 1-84.

16. S. Grosser and M. Moskowitz, On central topological groups, Trans. Amer. Math. Soc. 127 (1967), 317-340.

17. Compactness conditions in topological groups, J. Reine Agnew. Math. 246 (1971), 1-40.

18. A. Grothendieck, Critères de compacité dans les espaces functionnels généraux, Amer. J. Math. 74 (1952), 168-186.

19. E. Hewitt and K. A. Ross, Abstract harmonic analysis. II, Springer-Verlag, Berlin and New York, 1970.

20. J. M. López and K. A. Ross, Sidon sets, Marcel Dekker, New York, 1975.

21. P. Milnes, Almost periodic compactifications of direct and semidirect products, Colloq. Math. 44 (1981), 125-136.

22. M. A. Picardello, Lacunary sets in discrete noncommutative groups, Boll. Un. Mat. Ital. 8 (1973), 494-508.

23. D. Ramirez, Weakly almost periodic functions and Fourier-Stieltjes transforms, Proc. Amer. Math. Soc. 19 (1968), 1087-1088.

24. W. Rudin, Weak almost periodic functions and Fourier-Stieltjes transforms, Duke Math. J. 26 (1959), 215-220.

25. C. Ryll-Nardzewski, On fixed points of semigroups of endomorphisms of linear spaces, Proc. Fifth Berkeley Sympos. Math. Statist. Prob., Vol II, Part I, Theory of Probability, Univ. of California Press, Berkeley, 1966, pp. 55-61.

26. W. A. Veech, Weakly almost periodic functions on semisimple Lie groups, Monatsh. Math. 88 (1979), 55-68.

Department of Mathematics, State University of New York at Buffalo, Buffalo, New York 14214 